\title{
GENERAL EMBEDDING PROPERTIES OF ABSOLUTE BOREL AND SOUSLIN SPACES
}

\author{
ROGER W. HANSELL
}

\begin{abstract}
AbSTRACr. In a recent paper, S. Willard established several characterizations of absolute metric $G_{\alpha}$-spaces in terms of the Borel character they possessed as subspaces of certain compact Hausdorff spaces; and he asks whether a similar result holds for the $F_{\alpha}$-spaces. In the present paper, we show that for a metric space $X$ the following are equivalent for $\alpha \geqq 2$ : (1) $X$ is an absolute metric $F_{\alpha}$-space, (2) $X$ is a $Z_{\alpha} \cap G_{\delta}$-set (i.e., a Baire set of class $\alpha$ intersected with a $G_{\delta}$-set) in some compactification, (3) $X$ is an $F_{\alpha} \cap G_{\delta}$ set in every completely regular Hausdorff embedding, (4) $X$ is an absolute $F_{\alpha}$-space with respect to the class of all perfectly normal spaces. These properties remain equivalent when " $F_{\alpha}$ " and " $Z_{\alpha}$ " are replaced by "Souslin." Necessary and sufficient conditions for a metric space to be an $F_{\alpha}$-set in all its compactifications are found and, throughout, extensions to spaces which are not necessarily metrisable are provided.
\end{abstract}

1. Introduction. If $\varepsilon$ is a family of subsets of a fixed set $X$ and $\alpha$ is a countable ordinal, we define the family $\varepsilon_{\alpha}$ as follows: $\varepsilon_{0}=\mathcal{E}$ and, assuming the families have been defined for all ordinals $<\alpha$, we define $\varepsilon_{\alpha}$ to be the family of all sets of the form $\bigcup_{n=1}^{\infty} A_{n}$ or $\bigcap_{n=1}^{\infty} A_{n}$ where each $A_{n}$ belongs to $\varepsilon_{\alpha_{n}}$ for some $\alpha_{n}<\alpha$. When $X$ is a topological space and $\varepsilon$ is the family of all open sets in $X$, respectively the family of all closed (zero) sets, it is customary to replace $\varepsilon_{\alpha}$ by $G_{\alpha}(X)$, respectively $F_{\alpha}(X)\left(Z_{\alpha}(X)\right)$, or simply $G_{\alpha}$, or $F_{\alpha}$ etc. if the space $X$ is understood. If $X$ is a metric space, then $U G_{\alpha}(X)=\bigcup F_{\alpha}(X)=\bigcup Z_{\alpha}(X)$, where the union is over all countable ordinals $\alpha$, and the sets belonging to this collection are called the Borel sets of $X$. We note that this classification of the Borel sets-into classes which form an increasing transfinite sequence of type $\omega_{1}$-differs slightly from the usual classification given for metric spaces (see e.g. [6, p. 345]). ${ }^{1}$ However, it is easily verified that the results established in this paper are equally valid with respect to the usual classification. A member of the family $\varepsilon_{\alpha}$ will be called an $\varepsilon_{\alpha}$-set in $X$.

Received by the editors February 16, 1970.

AMS 1969 subject classifications. Primary 0440, 5453; Secondary 5435.

Key words and phrases. Descriptive set theory, Borel classifications, Souslin schemes, absolute $F_{\alpha}$-spaces, absolute Souslin spaces, compactifications, metric spaces.

${ }^{1}$ For a given metric space, if we let $F_{\alpha}^{\prime}$ and $G_{\alpha}^{\prime}$ denote the families of sets of class $\alpha$ relative to the traditional classification scheme, then one easily sees that $F_{\alpha}=F_{\alpha}^{\prime}$ $\not G_{\alpha}^{\prime}=G_{\alpha}$ for finite $\alpha$, whereas $F_{\alpha}=F_{\alpha}^{\prime} \cup G_{\alpha}^{\prime}=G_{\alpha}$ for $\omega_{0} \leqq \alpha<\omega_{1}$. 
A topological space $X$ is said to be an absolute metric $G_{\alpha}$-set (abbreviated $A G_{\alpha}$ (metric)) provided $X$ is metrisable and is a $G_{\alpha}$-set in every metric space in which it is topologically embedded. Spaces which are $A F_{\alpha}$ (metric) are defined analogously. And, of course, the definitions can be applied, in an obvious way, to more general classes of spaces.

With this terminology, a classical result due in part to Alexandrov, Cech, and Sierpiński, states that the following properties of a metric space $X$ are equivalent: (1) $X$ is completely metrisable, (2) $X$ is a $G_{1}=G_{\delta}$-set in some complete metric space, (3) $X$ is a $G_{\delta}$-set in its Stone-Cech compactification $\beta X$, and (4) $X$ is an $A G_{\delta}$ (metric). Classical too is the theorem of M. Lavrentiev [6, p. 431] which states that properties (2) and (4) above remain equivalent when $G_{\delta}$ is replaced by $G_{\alpha}$ (or $F_{\alpha}$ ), for $\alpha \geqq 1$ (respectively $\alpha \geqq 2$ ). More recently, S. Willard in [9] generalized the equivalence of (3) and (4) as follows:

TheOREM (WILlaRd). For a metrisable space $X$, the following are equivalent for $\alpha \geqq 1$ :

(1) $X$ is an $A G_{\alpha}$ (metric),

(2) $X$ is a $G_{\alpha}$-set in $\beta X$,

(3) $X$ is a $G_{\alpha}$-set in some compactification of $X$.

Later in [10] the theorem was extended to include:

(4) $X$ is a $G_{\alpha}$-set in every compactification of $X$, and

(5) $X$ is a $G_{\alpha}$-set in its closure in every completely regular Hausdorff embedding.

The question was also raised: "Can a result similar to the theorem above be obtained for $F_{\alpha}$-sets?" (see [9, p. 324]). The purpose of this note is to provide the following analogue of Willard's theorem for $F_{\alpha^{-}}$ sets:

MAIN THEOREM. For a metrisable space $X$, the following conditions are equivalent for $\alpha \geqq 2$ :

(1) $X$ is an $A F_{\alpha}$ (metric),

(2) $X$ is a $Z_{\alpha} \cap G_{\delta}$-set (i.e., $a Z_{\alpha}$-set intersected with $a G_{\delta^{-}}$-set) in some compactification,

(3) $X$ is a $Z_{\alpha} \cap G_{\delta}$-set in $\beta X$,

(4) $X$ is an $F_{\alpha} \cap G_{\delta}$-set in every compactification,

(5) $X$ is an $F_{\alpha} \cap G_{\delta}$-set in every completely regular Hausdorff embedding,

(6) $X$ is an $A F_{\alpha}$ (perfectly normal spaces). ${ }^{2}$

${ }^{2}$ The referee has brought to my attention the paper by $Z$. Frolik [3] in which the equivalence of (1), (2), and (3) is stated (Theorem 3(1), p. 782) in a less precise form (without any reference to classes). The same theorem covers the equivalence of (1), (2), and (3) of our corollary to Theorem 3 in $\$ 5$. 
If, in addition, we assume in (1) that $X$ is separable, then the conditions remain equivalent with " $Z_{\alpha} \cap G_{\delta}$ " and " $F_{\alpha} \cap G_{\delta}$ " replaced by " $Z_{\alpha}$ " and " $F_{\alpha}$ " respectively (corollary to Theorem $2, \S 4$ ). Both theorems are first proven for a certain class of perfectly normal spaces which properly contains the class of all metrisable spaces (Theorems 1 and $2, \$ \S 3$ and 4$)$. Finally, analogues for absolute Souslin spaces are given (Theorems 3 and $4, \S 5$ ).

2. Preliminaries. All spaces considered are assumed to be completely regular and Hausdorff (abbreviated CR-T $T_{2}$ ) unless we state the contrary.

Let $X$ be a fixed space and suppose that to each finite sequence $\left(n_{1}, \cdots, n_{p}\right)$ of natural numbers there corresponds a closed subset $F\left(n_{1}, \cdots, n_{p}\right)$ of $X$. Then the set

$$
S=\bigcup_{\left(n_{p}\right)} \bigcap_{p=1}^{\infty} F\left(n_{1}, \cdots, n_{p}\right),
$$

where the union is over all sequences $\left(n_{p}\right)=\left(n_{1}, n_{2}, \ldots\right)$ of natural numbers, is called a Souslin set in $X[5$, p. 203]. By an absolute (metric) Souslin set we mean a (metric) space which is a Souslin set in every (metric) space in which it can be embedded. We now list several well-known properties of Borel and Souslin sets.

2.1 For all $E \subset X$, if $E$ is a $Z_{\alpha}$-set in $X$, then $E$ is an $F_{\alpha}$-set in $X$, and this in turn implies that $E$ is a Souslin set in $X$.

2.2 If $E \subset X \subset Y$, then $E$ is an $F_{\alpha}$-set ( $G_{\alpha}$-set, Souslin set) in $X$ if and only if it is of the form $X \cap M$ where $M$ has the corresponding property in $Y$.

2.3 The complement of a $G_{\alpha}$-set is an $F_{\alpha}$-set, and every $F_{\alpha}$-set (respectively $Z_{\alpha}$-set) is an $F_{\beta}$-set $\left(Z_{\beta}\right.$-set) for all $\beta \geqq \alpha$.

$2.4 \mathrm{~A}$ finite intersection or union of $F_{\alpha}$-sets (respectively $Z_{\alpha}$-sets, $G_{\alpha}$-sets) is again of that class.

2.5 If $f: X \rightarrow Y$ is continuous, and $E \subset Y$ is an $F_{\alpha}$-set $\left(Z_{\alpha}\right.$-set, $G_{\alpha}$-set, Souslin set) in $Y$, then $f^{-1}(E)$ has the same property with respect to $X$.

2.6 (Choquet [1]) Every Souslin set in a compact space is Lindelöf. We will also have need of the following basic lemma.

$2.7[4$, p. 92] If $f$ is a continuous mapping of a space $E$ into a space $Y$, whose restriction to a dense subspace $X$ is a homeomorphism, then $f(E-X) \subset Y-f(x)$.

3. Proof of Main Theorem. Let us first recall that a topological space $X$ is said to be complete in the sense of $\check{C}$ ech if $X$ is CR-T $T_{2}$ and satisfies one of the following three equivalent conditions [2, p. 142]: 
(i) $X$ is a $G_{\delta}$-set in some compactification,

(ii) $X$ is a $G_{\delta}$-set in $\beta X$,

(iii) $X$ is a $G_{\delta}$-set in every compactification.

Also, a space $X$ is called perfectly normal if it is normal and if every closed subset is a $G_{\delta}$-set; or equivalently, if every closed subset is a zero set (see [2, Problem 1.G, p. 60]). We single out those spaces $X$ which are densely embeddable in perfectly normal complete spaces in the sense of Cech. Every metrisable space has this property, since every metric space is perfectly normal [2, p. 175] and each is densely embeddable in some complete metric space, which is also complete in the sense of Cech (see $\$ 1$ ). ${ }^{3}$ We will prove that for such spaces $X$ our Main Theorem is valid. More precisely, we prove the following:

THEOREM 1. Let $X$ be densely embedded in a space $X^{*}$ which is perfectly normal and complete in the sense of Cech.

Then the following conditions are equivalent for $\alpha \geqq 2$ :

(1) $X$ is an $F_{\alpha^{-}}$-set in $X^{*}$,

(2) $X$ is a $Z_{\alpha} \cap G_{\delta}$-set in some compactification,

(3) $X$ is a $Z_{\alpha} \cap G_{\delta}$-set in $\beta X$,

(4) $X$ is an $F_{\alpha} \cap G_{\delta}$-set in every compactification,

(5) $X$ is an $F_{\alpha} \cap G_{\delta}$-set in every CR-T $T_{2}$ space in which it is embedded,

(6) $X$ is an $A F_{\alpha}$ (perfectly normal spaces).

Proof. (1) $\rightarrow$ (2). We show by transfinite induction that for every $F_{\alpha}$-set $F$ in $X^{*}$ there exists a $Z_{\alpha}$-set $Z$ in $\beta X^{*}$ such that $F=Z \cap X^{*}$. For $\alpha=0, F$ is a closed (and hence zero) set in $X^{*}$, say $F=f^{-1}(\{0\})$, where $f$ is a continuous bounded real-valued function on $X^{*}$. Let $f_{\beta}$ be the Stone extension of $f$ to $\beta X^{*}$ and set $Z=f_{\beta}^{-1}(\{0\})$. Then, clearly, $F=Z \cap X^{*}$, and so $Z$ is the desired zero set. Assuming the above is true for all ordinals $<\alpha(\alpha$ a countable ordinal $>0)$, let $A_{n}$ be an $F_{\alpha_{n}}$-set in $X^{*}, \alpha_{n}<\alpha, n=1,2, \cdots$. Accordingly, let $Z_{n}$ be a $Z_{\alpha_{n}}$-set in $\beta X^{*}$ such that $A_{n}=Z_{n} \cap X^{*}$. Then, if $F=\cup_{n=1}^{\infty} A_{n}, Z$ $=\bigcup_{n=1}^{\infty} Z_{n}$ is a $Z_{\alpha}$-set in $\beta X^{*}$ and $F=Z \cap X^{*}$. Similarly, if $F=\bigcap_{n=1}^{\infty} A_{n}$, we may take $Z=\bigcap_{n=1}^{\infty} Z_{n}$, and the induction is complete. It follows that if $X$ is an $F_{\alpha}$-set in $X^{*}$, then $X=Z \cap X^{*}$ for some $Z_{\alpha}$-set $Z$ in $\beta X^{*}$. Since $X^{*}$ is a $G_{\delta}$-set in $\beta X^{*}$ and $\beta X^{*}$ is a compactification of $X$, we have shown that (2) follows from (1).

${ }^{3}$ Not every space with this property is metrisable. The following example of a compact, perfectly normal but nonmetrisable space was suggested by the referee: Topologise the lexicographically ordered square with the order topology, and consider the closed, and hence compact, subspace formed by the top and bottom edges. One then shows, with some difficulty, that this subspace is perfectly normal but not second countable (and hence nonmetrisable). 
$(2) \rightarrow(3)$. Let $X=Z \cap G$ where $Z$ is a $Z_{\alpha}$-set and $G$ is a $G_{\delta}$-set in some fixed (but arbitrary) compactification $b X$ of $X$. Let $f: \beta X \rightarrow b X$ be the Stone extension of the embedding $X \rightarrow b X$. By $2.5, f^{-1}(Z \cap G)$ $=f^{-1}(Z) \cap f^{-1}(G)$ is a $Z_{\alpha} \cap G_{\delta}$-set in $\beta X$; and, by $2.7, f^{-1}(Z \cap G)=X$. Hence (3). The more difficult proof that $(3) \rightarrow(4)$ requires a lemma.

LEMMA 1. Let $X$ be a CR- $T_{2}$ space and let $b X$ be a compactification of $X$. Let $f: \beta X \rightarrow b X$ be the Stone extension of the embedding $X \rightarrow b X$ and let $\tau$ denote the topology on the set $\beta X$ consisting of all sets of the form $f^{-1}(W)$ where $W$ is an open subset of $b X$ (note that $(\beta X, \tau)$ is compact but not necessarily CR- $T_{2}$ ).

(a) If $U$ is open in $\beta X$ and $V=\operatorname{Int}_{r}(U)$, then $U \cap X=V \cap X$.

(b) If $Z$ is a zero set in $\beta X$, then $\mathrm{Cl}_{r}(Z \cap X) \cap X=Z \cap X$, and there exists a $G_{8}$-set $G$ in $(\beta X, \tau)$ such that $X \subset G$ and $\mathrm{Cl}_{r}(Z \cap X) \cap G \subset Z$.

(c) $A$ necessary and sufficient condition for $E \subset \beta X$ to be an $F_{\alpha}$-set (respectively $G_{\alpha}$-set, Souslin set) in $(\beta X, \tau)$, is that it be of the form $f^{-1}(M)$ where $M$ has the corresponding property in $b X$.

Proof. To prove (a), it is enough to show that $U \cap X \subset V \cap X$, so let $x \in U \cap X$. Since $f(\beta X-X) \subset b X-X$ by 2.7 , the set $f(\beta X-U)$ $=f(\beta X-X) \cup f(X-U)$ does not contain $f(x)$. Thus $f(x) \in b X$ $-f(\beta X-U)$, from which it follows that

$$
x \in f^{-1}(b X-f(\beta X-U))=\beta X-f^{-1}(f(\beta X-U)) \subset U .
$$

Since $\beta X-U$ is compact, $f(\beta X-U)$ is compact, and so $b X$ $-f(\beta X-U)$ is open in $b X$. Thus we have shown that $x \in \operatorname{Int}_{\tau}(U)$ $\cap X=V \cap X$.

To prove (b), note first that zero sets are $G_{\delta}$-sets $[2$, p. 15], so $Z=\bigcap_{n=1}^{\infty} U_{n}$ where each $U_{n}$ is open in $\beta X$. Let $V_{n}=$ Int,$U_{n}$ and observe that, by (a) above, $Z \cap X=\left(\bigcap_{n=1}^{\infty} V_{n}\right) \cap X$. Now put $G=\left(\beta X-\mathrm{Cl}_{\tau}(Z \cap X)\right) \cup\left(\cap_{n=1}^{\infty} V_{n}\right)$. It follows from 2.4 that $G$ is a $G_{\delta}$-set with respect to $(\beta X, \tau)$. Since the $\tau$ subspace topology on $X$ coincides with its original topology, we have $\mathrm{Cl}_{\tau}(Z \cap X) \cap X=Z \cap X$, and thus $\beta X-\mathrm{Cl}_{\tau}(Z \cap X) \supset X-(Z \cap X)$. This, together with the fact that $\bigcap_{n=1}^{\infty} V_{n} \supset Z \cap X$, proves that $G \supset X$. Finally, we have $\mathrm{Cl}_{\tau}(Z \cap X)$ $\cap G=\mathrm{Cl}_{\tau}(Z \cap X) \cap\left(\bigcap_{n-1}^{\infty} V_{n}\right) \subset Z$, as follows easily from the definition of $G$ and the fact that $\bigcap_{n=1}^{\infty} V_{n} \subset Z$. This completes the proof of (b).

That the condition in (c) is sufficient follows immediately from property 2.5. On the other hand, the necessity of the condition follows easily from the fact that a set $E$ is $\tau$-closed (resp. $\tau$-open) in $\beta X$ if and only if it is of the form $f^{-1}(M)$ where $M$ is closed (resp. open) in $b X$, and the fact that the inverse image operator preserves intersections and unions. The routine induction arguments are omitted. This completes the proof of Lemma 1. 
We now turn to the proof that $(3) \rightarrow(4)$. Let $X$ be a space, and let $Z$ be a $Z_{\alpha}$-set and $H$ and $G_{\delta}$-set, both with respect to $\beta X$, such that $X=Z \cap H$. Let $b X, f$, and $\tau$ have the same meaning attached to them in Lemma 1. We will show first that $X$ is an $F_{\alpha} \cap G_{\delta}$-set with respect to $(\beta X, \tau)$ (cf. the strategy used in [9]). Since $H$ is a $G_{\delta}$-set, $H$ $=\bigcap_{n=1}^{\infty} H_{n}$ where each $H_{n}$ is open in $\beta X$. Let $W_{n}=\operatorname{Int}_{\tau} H_{n}, n=1$, $2, \cdots$ Then, from (a) of Lemma $1, X=Z \cap W$ where $W=\bigcap_{n=1}^{\infty} W_{n}$.

We now assert that given any $Z_{\alpha}$-set $Z$ in $\beta X$ there exists an $F_{\alpha}$-set $F$ and a $G_{\delta}$-set $G$, both with respect to $(\beta X, \tau)$, such that $X \subset G$, $F \cap G \subset Z$, and $F \cap X=Z \cap X$. For $\alpha=0, Z$ is a zero set in $\beta X$, and so the existence of sets $F$ and $G$ is an immediate consequence of Lemma 1 part (b). Assuming the proposition has been shown for all ordinals $<\alpha(\alpha$ a countable ordinal $>0)$, let $Z=\bigcup_{n=1}^{\infty} A_{n}$ where $A_{n}$ is a $Z_{\alpha_{n}}$ set, $\alpha_{n}<\alpha, n=1,2, \cdots$. By the induction hypothesis, there exists $F_{\alpha_{n}}$-sets $F_{n}$ and $G_{\delta}$-sets $G_{n}$, with respect to $(\beta X, \tau)$, such that $X \subset G_{n}$, $F_{n} \cap G_{n} \subset A_{n}$, and $F_{n} \cap X=A_{n} \cap X$, for each $n$. Now set $F=\bigcup_{n=1}^{\infty} F_{n}$ and $G=\bigcap_{n=1}^{\infty} G_{n}$. Then $F$ is an $F_{\alpha}$-set and $G$ is a $G_{\delta}$-set in $(\beta X, \tau)$, $X \subset G, \quad F \cap G \subset \bigcup_{n=1}^{\infty} F_{n} \cap G_{n} \subset \bigcup_{n=1}^{\infty} A_{n}=Z$, and $F \cap X=\bigcup_{n=1}^{\infty} F_{n} \cap X$ $=\bigcup_{n=1}^{\infty} A_{n} \cap X=Z \cap X$. Similarly, if $Z=\bigcup_{n=1}^{\infty} A_{n}$, we may take $F=\bigcap_{n=1}^{\infty} F_{n}$ and $G=\bigcap_{n=1}^{\infty} G_{n}$, and so the assertion is proved.

Returning to the equation $X=Z \cap W$, let $F$ and $G$ satisfy the above assertion for $Z$. Then $X=Z \cap X=F \cap X \subset F$, and so we have $X \subset F \cap G \cap W$. On the other hand, $(F \cap G) \cap W \subset Z \cap W=X$. Since $F$ is an $F_{\alpha}$-set and $G \cap W$ is a $G_{\delta}$-set in $(\beta X, \tau)$, we have shown that $X=F \cap(G \cap W)$ is an $F_{\alpha} \cap G_{\delta}$-set in $(\beta X, \tau)$. Now, by (c) of Lemma 1 , there exists an $F_{\alpha}$-set $F^{\prime}$ and a $G_{\delta}$-set $H^{\prime}$ in $b X$ such that $F=f^{-1}\left(F^{\prime}\right)$ and $G \cap W=f^{-1}\left(H^{\prime}\right)$. Consequently, $X=f^{-1}\left(F^{\prime} \cap H^{\prime}\right)$. But, by property 2.7, $X=f(X)$, and so we have $X=F^{\prime} \cap H^{\prime}$, proving that $X$ is an $F_{\alpha} \cap G_{\delta}$-set in $b X$. This completes the proof of the implication $(3) \rightarrow(4)$.

$(4) \rightarrow(5)$. Assume $X$ satisfies (4) and let $X$ be a subspace of the $\left(\mathrm{CR}-T_{2}\right)$ space $Y$. Then $\beta\left(\mathrm{Cl}_{Y} X\right)$ is a compactification of $X$, so $X$ is an $F_{\alpha} \cap G_{\delta}$-set in $\beta\left(\mathrm{Cl}_{Y} X\right)$. From 2.2 (\$2), we conclude that $X$ has the same property in $\mathrm{Cl}_{Y} X$. Hence, by another application of $2.2, X$ is of the form $(F \cap H) \cap C_{Y} X$ where $F \cap H$ is an $F_{\alpha} \cap G_{\delta}$-set in $Y$. It follows that $X=\left(F \cap \mathrm{Cl}_{Y} X\right) \cap H$ is an $F_{\alpha} \cap G_{\delta}$-set in $Y$. Moreover, if $Y$ is also perfectly normal, then $H$ is an $F_{\sigma \delta}$-set; thus, since $\alpha \geqq 2, X$ is an $F_{\alpha}$-set in $Y$. Hence we have shown that $(4) \rightarrow(5) \rightarrow(6)$.

Since the implication $(6) \rightarrow(1)$ is trivial, all the equivalences in Theorem 1 have been established.

Our Main Theorem is, of course, an immediate consequence of Theorem 1 ; we need only take for $X^{*}$ any metric completion of $X$. 
REMARK. It would be interesting to know whether or not " $Z_{\alpha} \cap G_{\delta^{-}}$ set" can be replaced by " $F_{\alpha} \cap G_{\delta}$-set" in conditions (2) and (3) of Theorem 1. On the other hand, can one characterize absolute $Z_{\alpha}$-sets by replacing $F_{\alpha}$ by $Z_{\alpha}$ in statements (1), (4), (5), and (6) of Theorem 1? (Cf. [7, Remark 2.2].)

4. It is natural at this juncture to ask "When is a space an $F_{\alpha}$-set in all of its compactifications?" The answer takes a surprisingly simple form.

TheORem 2. Let $X$ be densely embeddable in a space $X^{*}$ which is perfectly normal and complete in the sense of Cech. Then the following conditions are equivalent for $\alpha \geqq 2$ :

(1) $X$ is a Lindelöf space and an $A F_{\alpha}\left(\mathrm{CR}-T_{2}\right)$,

(2) $X$ is a Lindelöf space and an $F_{\alpha}$-set in $X^{*}$,

(3) $X$ is a $Z_{\alpha}$-set in some compactification,

(4) $X$ is a $Z_{\alpha}$-set in $\beta X$,

(5) $X$ is an $F_{\alpha^{-}}$set in every compactification,

(6) $X$ is an $A F_{\alpha}\left(\mathrm{CR}-T_{2}\right)$.

REMARK. The equivalence of (3) and (4) is essentially known (see [7, Theorem 2.6, p. 692]).

We first prove a basic lemma.

LemMa 2. If $X$ is a Lindelöf space and is an $F_{\alpha} \cap G_{\delta^{-}}$set (or a $Z_{\alpha} \cap G_{\delta^{-}}$ set) in some $\mathrm{CR}-T_{2}$ space $Y$, then $X$ is an $F_{\alpha}$-set $\left(Z_{\alpha}\right.$-set) in $Y$ provided $\alpha \geqq 2$.

Proof. Suppose $X=F \cap H$ where $F$ is an $F_{\alpha}$-set $\left(Z_{\alpha}\right.$-set) and $H$ is a $G_{\delta}$-set in $Y$. Let $H=\bigcap_{n=1}^{\infty} H_{n}$, where each $H_{n}$ is open in $Y$. Since $Y$ is CR-T $T_{2}$, we can cover $X$, for each $n$, by interiors of zero-sets $\left\{Z_{x_{n}}\right\}$ in $Y$ such that $Z_{x n} \subset H_{n}$ for each $x \in X[4, \S 3.2]$. Since $X$ is Lindelöf, $X$ is contained in the union $Z_{n}$ of a countable subcollection of the $Z_{x n}$. It follows that $\bigcap_{n=1}^{\infty} Z_{n}$ is a $Z_{2}$-set in $Y$, and that

$$
X \subset F \cap\left(\bigcap_{n=1}^{\infty} Z_{n}\right) \subset F \cap\left(\bigcap_{n=1}^{\infty} H_{n}\right)=F \cap H=X .
$$

Therefore, $X=F \cap\left(\bigcap_{n=1}^{\infty} Z_{n}\right)$ and hence, since $\alpha \geqq 2$, is an $F_{\alpha}$-set (respectively, a $Z_{\alpha}$-set) in $Y$.

To prove Theorem 2 we note first that $(1) \rightarrow(2)$ since $X^{*}$ is CR-T $T_{2}$. Assuming (2), it follows from Theorem 1 that $X$ is a $Z_{\alpha} \cap G_{\delta}$-set in some compactification, and hence, by the lemma, that $X$ is a $Z_{\alpha}$-set in the same compactification. Hence (2) $\rightarrow(3)$. If $X$ is a $Z_{\alpha}$-set in $b X$, a compactification of $X$, then surely $X$ is a $Z_{\alpha} \cap G_{\delta}$-set in $b X$. And 
so, by Theorem 1, $X$ is a $Z_{\alpha} \cap G_{\delta}$-set in $\beta X$ and hence a $Z_{\alpha}$-set in $\beta X$, by Lemma 2 (which applies in view of properties 2.1 and 2.6). Thus $(3) \rightarrow(4)$. Similarly, using Theorem 1 and Lemma 2, we deduce that $(4) \rightarrow(5)$ and $(5) \rightarrow(6)$. Again, $(6) \rightarrow(1)$ by 2.1 and 2.6 of $\$ 2$. This completes the proof of Theorem 2 .

Corollary. For a metrisable space $X$, the following conditions are equivalent for $\alpha \geqq 2$ :

(1) $X$ is separable and an $A F_{\alpha}$ (metric),

(2) $X$ is a $Z_{\alpha}$-set in some compactification,

(3) $X$ is $a Z_{\alpha}$-set in $\beta X$,

(4) $X$ is an $F_{\alpha^{-}}$set in every compactification,

(5) $X$ is an $A F_{\alpha}\left(\mathrm{CR}-T_{2}\right)$.

Proof. Since all metrisable spaces satisfy the hypothesis of Theorem 2, the equivalence of (2) through (5) follows immediately from the theorem. On the other hand, since a metric space is separable if and only if it is Lindelöf, it is clear from Theorem 2 that (5) $\rightarrow(1)$. Moreover, (1) implies that $X$ is an $F_{\alpha}$-set, and hence a $Z_{\alpha}$-set, in the compact space $I^{\aleph_{0}}$ (the Hilbert cube), and so $(1) \rightarrow(2)$. The proof is thus complete.

REMARK. For an interesting discussion of $A F_{\alpha}$ (metric) spaces for $\alpha<2$, the reader is referred to the paper of Stone [8].

5. Absolute Souslin sets. We prove here analogues of Theorems 1 and 2 for absolute Souslin sets. We first state as a lemma the following fact proved in $[1$, p. 78].

Lemma (Choquet). If $f: X \rightarrow Y$ is a continuous mapping between two compact spaces and $S \subset X$ is a Souslin set in $X$, then $f(S)$ is a Souslin set in $Y$.

Theorem 3. Let $X$ be densely embeddable in a space $X^{*}$ which is perfectly normal and complete in the sense of Cech. Then the following conditions are equivalent:

(1) $X$ is a Souslin set in $X^{*}$,

(2) $X$ is a Souslin set intersected with a $G_{\delta}$-set in some compactification,

(3) $X$ is a Souslin set intersected with a $G_{\delta}$-set in $\beta X$,

(4) $X$ is a Souslin set intersected with $a G_{b}$-set in every compactification,

(5) $X$ is a Souslin set intersected with a $G_{\delta}$-set in every CR-T embedding,

(6) $X$ is an absolute Souslin set in the class of all perfectly normal spaces. 
Proof. (1) $\rightarrow$ (2). If $X$ is a Souslin set in $X^{*}$, then, by $2.2, X$ is of the form $S \cap X^{*}$, where $S$ is a Souslin set in $\beta X^{*}$. Since $X^{*}$ is a $G_{\delta}$-set in $\beta X^{*}$, the implication is proved.

$(2) \rightarrow(3)$. Let $X=S \cap G$ where $S$ is a Souslin set and $G$ is a $G_{\delta}$-set in $b X$, a compactification of $X$. If $f: \beta X \rightarrow b X$ denotes the Stone extension of the embedding $X \rightarrow b X$, then, by $2.5, f^{-1}(S \cap G)=f^{-1}(S)$ $\cap f^{-1}(G)$ is a Souslin set intersected with a $G_{\delta}$-set in $\beta X$. However, by $2.7, f^{-1}(S \cap G)=X$, proving (3).

$(3) \rightarrow(4)$. Again, suppose that $X=S \cap G$, where $S$ is a Souslin set and $G$ is a $G_{\delta}$-set in $\beta X$, and let $b X$ be a given compactification of $X$. Letting $f$ denote again the Stone extension of the embedding $X$ $\rightarrow b X$, there exists, by parts (a) and (c) of Lemma 1, a $G_{\delta}$-set $H$ in $b X$ such that $X=S \cap f^{-1}(H)$ (cf. the proof of the corresponding part in Theorem 1). Hence $f(X)=f(S) \cap H$. By the preceding lemma, $f(S)$ is a Souslin set in $b X$, and $X=f(X)$ by 2.7. It follows that $X$ is a Souslin set intersected with a $G_{\delta}$-set in $b X$.

The proofs that $(4) \rightarrow(5),(5) \rightarrow(6)$, and $(6) \rightarrow(1)$ are entirely analogous to the proofs given for the corresponding implications in Theorem 1, and so we omit the details. This completes the proof of Theorem 3.

Corollary. For a metrisable space $X$, conditions (2)-(6) of Theorem 3 are all equivalent to:

(1) $X$ is an absolute metric Souslin set. ${ }^{2}$

The proof of our final theorem and corollary differs in no essential way from the proofs given already for Theorem 2 and its corollary. The reader should have no trouble in supplying the details.

TheOREM 4. Let $X$ be densely embeddable in a space $X^{*}$ which is perfectly normal and complete in the sense of Cech. Then the following conditions are equivalent:

(1) $X$ is a Lindelö space and an absolute Souslin set for the class of all CR- $T_{2}$ spaces,

(2) $X$ is a Lindelöf space and a Souslin set in $X^{*}$,

(3) $X$ is a Souslin set in some compactification,

(4) $X$ is a Souslin set in $\beta X$,

(5) $X$ is a Souslin set in every compactification,

(6) $X$ is an absolute Souslin set for the class of all CR-T spaces.

CoRollary. For a metrisable space $X$, conditions (3)-(6) of Theorem. 4 are all equivalent to:

(1) $X$ is separable and an absolute metric Souslin set. 
The author wished to express his appreciation to Professor A. H. Stone for having brought the above problem to his attention, and for many helpful comments.

\section{REFERENCES}

1. G. Choquet, Ensembles $\mathcal{K}$-analytiques et $\mathcal{K}$-sousliniens. Cas gênéral et cas métrique, Ann. Inst. Fourier (Grenoble) 9 (1959), 75-81. MR 22 \#3692a.

2. R. Engelking, Outline of general topology, PWN, Warsaw, 1965; English transl., North-Holland, Amsterdam; Interscience, New York, 1968. MR 36 \#4508; MR 37 \#5836.

3. Z. Frolik, $A$ note on $C(P)$ and Baire sets in compact and metrizable spaces, Bull. Acad. Polon. Sci. Sér. Sci. Math. Astronom. Phys. 15 (1967), 779-784. MR 37 \#79.

4. L. Gillman and M. Jerison, Rings of continuous functions, University Series in Higher Math., Van Nostrand, Princeton, N. J., 1960. MR 20 \#6994.

5. F. Hausdorff, Set theory, 2nd ed., de Gruyter, Berlin, 1935; English transl., Chelsea, New York, 1962. MR 25 \#4999.

6. K. Kuratowski, Topologie. Vol. I, PWN, Warsaw, 1958; English transl., Academic Press, New York; PWN, Warsaw, 1966. MR 19, 873.

7. S. Negrepontis, Absolute Baire sets, Proc. Amer. Math. Soc. 18 (1967), 691694. MR $35 \# 4883$.

8. A. H. Stone, Absolute $F_{\sigma}$-spaces, Proc. Amer. Math. Soc. 13 (1962), 495-499. MR 25 \#1535; errata, MR 25 p. 1242.

9. S. Willard, Absolute Borel sets in their Stone-Čech compactifications, Fund. Math. 58 (1966), 323-333. MR 33 \#4892.

10. - Embedding metric absolute Borel sets in completely regular spaces, Colloq. Math. 20 (1969), 83-88. MR 39 \#3458.

University of Connecticut, Storrs, Connecticut 06268 